\title{
Clima de seguridad laboral y conductas de seguridad en una empresa de la industria del acero en el Perú
}

MANuel Enrique Herrera Díaz ${ }^{1}$

\begin{abstract}
RESUMEN
Los trabajadores son el eje principal de la seguridad y salud en el trabajo, razón por la cual las empresas deben orientar sus esfuerzos hacia conocer cada vez más a su capital humano y lograr su toma de conciencia y participación en la gestión preventiva de la seguridad. Las variables utilizadas en la presente investigación son el clima de seguridad laboral (CLS), las conductas de seguridad (COS) y variables sociolaborales. Se verificó la fiabilidad mediante el alfa de Cronbach y las relaciones entre las variables se evaluaron con el coeficiente de Spearman, las pruebas $t$ y ANOVA. Los principales resultados obtenidos son: 1) la fiabilidad de los constructos, 2) la verificación de la correlación significativa y positiva entre CLS y COS, 3) no se encontraron relaciones de causalidad de las variables sociolaborales con el CLS y las COS, y 4) las variables sociolaborales edad y tiempo de servicio influyen en la participación en la seguridad (PAS).
\end{abstract}

Palabras clave: clima de seguridad laboral; conductas de seguridad; cumplimiento de la seguridad; participación en la seguridad.

\section{INTRODUCCIÓN}

El pilar de la seguridad y salud en el trabajo (SST) es la prevención, a partir de ello se han desarrollado diferentes modelos y sistemas de gestión, fortalecidos por leyes nacionales y convenios internacionales; pero con el transcurso del tiempo se ha evidenciado que no han sido suficientes para contrarrestar los accidentes laborales y optimizar la seguridad en las organizaciones. Los sistemas preventivos resultan más efectivos cuando buscan la participación y la toma de conciencia de sus colaboradores, con miras a desarrollar una cultura preventiva de la seguridad. Por otro lado, muchas veces, la seguridad compite con otros intereses de la organización, como calidad y productividad, situación que puede provocar un debilitamiento de las conductas seguras de los trabajadores, perder el enfoque preventivo y originar accidentes laborales, entre otras consecuencias. Es en este sentido que cobra vital importancia monitorear el entorno laboral y las conductas de sus colaboradores, a fin de detectar riesgos tempranamente y convertirlos en oportunidades de mejora. El presente estudio contempla dos variables que apuntan a mejorar y fortalecer la gestión de la seguridad en el trabajo, como son el clima de seguridad laboral y las conductas de seguridad de los colaboradores.

La complejidad y amplitud del clima organizacional amerita mayor especificidad para fines puntuales $y$, de esta manera, tener medidas de climas laborales específicos. La característica del clima organizacional de actuar como antecedente de la conducta de los colaboradores convierte al clima de seguridad en una herramienta importante respecto a las conductas de los trabajadores en materia de seguridad y salud laboral. En 1980, Dov Zohar acuñó el concepto de clima de seguridad; observó que los factores organizacionales afectan los resultados relacionados con la seguridad, y enfatizó la importancia de considerar climas específicos como el clima de seguridad de una organización, para lo que estableció una distinción entre medidas globales y

1 Ingeniero electrónico, con especialización en administración de empresas, tecnologías de la información y comunicaciones. Es consultor independiente en gestión empresarial, gestión de operaciones, cadena de suministro, seguridad y salud ocupacional. (Lima, Perú). ORCID: https://orcid.org/0000-0002-4759-9554

E-mail: menriqueherrera@gmail.com 
medidas particulares del clima. Es así que el clima de seguridad se define como las percepciones que los trabajadores comparten sobre los aspectos vinculados a la seguridad en su trabajo, con hincapié en las actitudes y conductas de los supervisores y directivos (Zohar, 1980). El clima de seguridad evalúa las percepciones compartidas de los trabajadores en relación con las políticas (nivel estratégi$\mathrm{co}$ ), los procedimientos (nivel táctico) y las prácticas de seguridad (nivel operativo) en la organización y la comprensión del impacto que la empresa tiene sobre la conducta o desempeño de seguridad de sus trabajadores (Díaz, 2006).

Zohar y Luria (2005) desarrollaron el modelo de clima de seguridad multinivel MSC (Multilevel Safety Climate), que se centra en las políticas, los procedimientos y las prácticas de gestión como fuentes primarias o referentes de las percepciones climáticas de seguridad. Plantearon un clima de seguridad global a nivel de la organización y un clima más específico a nivel de grupo, donde las políticas y los procedimientos proporcionan referentes a nivel de organización, mientras que su implementación diaria por parte de los supervisores de primera línea proporciona referentes a nivel de grupo. Los autores crearon un instrumento para medir el clima de seguridad a nivel de grupo a través de un cuestionario de 16 ítems, con un formato de respuesta tipo Likert-5, con el cual obtuvieron una confiabilidad muy alta de la escala con un alfa de Cronbach de 0.95. Asimismo, determinaron fuertes correlaciones entre la carga de los factores mayores que 0.80 mediante un único factor para este clima de seguridad y la validez del constructo.

Por su parte, Johnson (2007) realizó un estudio para verificar el carácter predictivo del constructo «clima de seguridad» respecto a los resultados de seguridad (comportamiento, accidentes, etc.); para ello, aplicó el modelo MSC y el cuestionario de clima de seguridad de Zohar y Luria (2005) a 292 empleados en 20 compañías manufactureras. Mediante un modelo de ecuaciones estructurales, encontró fuerte correlación entre factores, mayor a 0.95 , con lo que demostró y reforzó lo propuesto por Zohar y Luria, indicando que la estructura de un solo factor proporciona un ajuste adecuado a los datos y representa el compromiso o prioridad de la administración hacia la seguridad. El autor concluye que: a) el cuestionario del modelo multinivel de clima de seguridad (MSC) es psicométricamente confiable y válido, y b) sirve como un efectivo predictor de los resultados de seguridad (comportamiento y accidentes).
En esta línea, Neal y Griffin (2004) argumentaron que la relación entre el clima de seguridad y los accidentes laborales está mediada por el comportamiento de seguridad. Es decir, el comportamiento de seguridad es el predictor más próximo a los accidentes laborales.

El clima de seguridad laboral se conceptualiza como: a) percepción subjetiva que tienen los trabajadores de la organización, sus miembros, su estructura y sus procesos; que b) a pesar de las diferencias individuales, presenta apreciaciones comunes; c) que se basa en elementos objetivos del ambiente o indicios, y que, además, d) se comporta como antecedente de la conducta de los trabajadores, cualidad que le otorga su verdadera importancia (Peiró, 1984; Coyle, Sleeman y Adams, 1995; Melià y Sesé, 1999).

A partir de la teoría del desempeño laboral en el campo de la seguridad ocupacional, se desarrolló el modelo bidimensional de las conductas seguras. Sus principales impulsores son Griffin y Neal (2000) y su modelo se basó en dos dimensiones: cumplimiento de la seguridad y participación en la seguridad. El cumplimiento de la seguridad contempla las tareas que los trabajadores deben realizar de forma inevitable a fin de mantener la seguridad en el lugar de trabajo. Estas actividades se desarrollan sujetas a las normas y procedimientos enmarcados en la prevención de la SST. La participación en la seguridad describe comportamientos que contribuyen indirectamente con la seguridad individual y colectiva en la organización, los cuales ayudan a desarrollar un entorno seguro, es decir, apoyan a la seguridad en el amplio contexto organizacional. Estos comportamientos están orientados a conductas voluntarias de los colaboradores.

En esta investigación se evaluó la variable conductas de seguridad (variable dependiente en este estudio) y sus dos dimensiones, el cumplimiento de seguridad y la participación en seguridad, con base en la escala de Neal y Griffin (2006), a través de seis afirmaciones. El estudio original de estos autores obtuvo una confiabilidad $\alpha=0.93$ para la dimensión cumplimiento de seguridad y $\alpha=0.89$ para la dimensión participación en seguridad. Las dos dimensiones resultaron estar altamente correlacionadas a nivel de grupo, por lo que se formula un solo indicador para las conductas de seguridad y se confirma, así, su validez de constructo $(r=0.91$, $p=0.001$ ).

El presente estudio se llevó a cabo en una empresa de la industria del acero en el Perú. En lo sucesivo de este documento, se utilizan los acrónimos 
siguientes: clima de seguridad laboral (CLS), conductas de seguridad (COS), cumplimiento de la seguridad (CUS) y participación en la seguridad (PAS).

\section{Hipótesis y variables}

$\mathrm{H}_{1}$ : Existe entre el clima de seguridad laboral (CLS) con las conductas de seguridad (COS) de los trabajadores del área de operaciones de una empresa de la industria del acero en el Perú.

$\mathrm{H}_{2}$ : Existe relación entre el clima de seguridad laboral (CLS) con el cumplimiento de la seguridad (CUS) en los trabajadores del área de operaciones de una empresa de la industria del acero en el Perú.

$\mathrm{H}_{3}$ : Existe relación entre el clima de seguridad laboral (CLS) con la participación en la seguridad (PAS) en los trabajadores del área de operaciones de una empresa de la industria del acero en el Perú.

$\mathrm{H}_{4}$ : Existe relación causal de las variables sociolaborales con el clima de seguridad laboral (CLS) en los trabajadores del área de operaciones de una empresa de la industria del acero en el Perú.

$\mathrm{H}_{5}$ : Existe relación causal de las variables sociolaborales con las conductas de seguridad (COS) en los trabajadores del área de operaciones de una empresa de la industria del acero en el Perú.

Asimismo, para el contraste de las relaciones causales, se consideraron como variables independientes las variables sociolaborales en $\mathrm{H}_{4} \mathrm{y} \mathrm{H}_{5}$, y como variables dependientes a CLS en $\mathrm{H}_{4}$ y COS, CUS y PAS en $\mathrm{H}_{5}$.

\section{METODOLOGÍA}

El enfoque de la investigación es cuantitativo, porque mide las variables tanto de clima de seguridad como de conductas de seguridad en función a su magnitud y, luego, se examinan los datos de manera numérica utilizando herramientas estadísticas con un diseño no experimental. El método usado es transversal-descriptivo y correlacional. Es transversal porque se recolectarán datos en un solo momento y un tiempo único; y es descriptivo y correlacional porque el propósito es describir tanto al CLS como a las COS y analizar su interrelación. Es causal cuando se analizan las variables sociolaborales con CLS, COS, CUS y PAS.

\section{Participantes}

El estudio se centra en los trabajadores del área de operaciones de una empresa de la industria del acero en el Perú. El área de operaciones desarrolla dos actividades principales: a) tratamiento térmico del acero y b) almacenamiento y corte de materiales de acero. El tratamiento térmico es aplicado a piezas de acero con la finalidad de mejorar sus propiedades mecánicas, especialmente la dureza, la resistencia y la elasticidad, mediante la aplicación de diferentes procesos como temple, revenido, cementación, nitruración, recocido, etc., en los que se hace uso de altas temperaturas. En las operaciones de almacenamiento y corte, los operarios manipulan y cortan, principalmente, barras y planchas de acero muy pesadas y de gran tamaño. El estudio se realizó con el total de trabajadores del área de operaciones, incluyendo al personal de gestión, es decir, con 42 trabajadores.

\section{Instrumento de medición}

Para medir el CLS se utilizó el modelo multinivel de seguridad propuesto por Zohar y Luria (2005), cuyo constructo consta de 16 ítems; para las COS, se realizó la medición a través de la escala propuesta por Neal y Griffin (2006), conformada por seis afirmaciones, donde las tres primeras miden la dimensión CUS y las tres restantes, la dimensión PAS. Se aplicaron cuestionarios con formato de respuesta tipo Likert-5 con las opciones siguientes: 1 = totalmente en desacuerdo, 2 = en desacuerdo, 3 = ni de acuerdo ni en desacuerdo, $4=$ de acuerdo y $5=$ totalmente de acuerdo.

Las variables CLS, COS, CUS y PAS son cualitativas-ordinales y sus rangos de valores son los siguientes: CLS, de 16 a 80; COS, de 6 a 30; CUS y PAS, de 3 a 15. Para mejor comprensión, los valores obtenidos en los resultados se expresaron en términos de porcentaje, a partir del valor máximo y mínimo que podría tomar cada variable, también, expresados en niveles, propuestos por el autor de este estudio, con los siguientes rangos: pésimo $(P)$, $\leq 50 \%$; muy bajo (MB), de $51 \%$ a $60 \%$; bajo $(B)$, de $61 \%$ a $70 \%$; intermedio (I), de $71 \%$ a $80 \%$; alto $(A)$, de $81 \%$ a $90 \%$; y muy alto (MA), de $91 \%$ a $100 \%$.

\section{Procedimiento}

La aplicación de los instrumentos de medición se realizó durante dos semanas. Se contó con el apoyo de la gerencia general, y la participación del área de recursos humanos y del área de seguridad laboral. Las respuestas se hicieron de manera anónima por grupos de trabajadores, todo coordinado previamente con las áreas antes indicadas y supervisado por un especialista. En promedio, la evaluación fue de 30 minutos. Luego, los datos obtenidos fueron procesados utilizando el software estadístico SPSS (versión 25). 


\section{RESULTADOS}

\section{Análisis descriptivo}

A través del análisis de las variables sociolaborales, se logró tener mayor conocimiento de la población participante en el presente estudio. Es así que las variables sociolaborales consideradas son sexo, edad, grado de instrucción, turno, puesto laboral, condición laboral contractual y tiempo de servicio en la empresa (experiencia). Del total de trabajadores, el $95 \%$ son hombres, y el $5 \%$, mujeres. La edad va desde 22 a 64 años, con una media de 38 años; y para el estudio se agruparon en tres categorías ( $\leq 30$, de 31 a $45 \mathrm{y} \geq 46$ años). El tiempo de servicio de los trabajadores en la empresa (en años) tiene una media de 10 y un rango de 1 a 47 años, y también fue agrupado en tres categorías $(\leq 1$, de 2 a 14 y $\geq 15$ ). Respecto al grado de instrucción, la distribución es: primaria $12 \%$, secundaria $57 \%$ y superior $31 \%$. Los operarios de tratamiento del acero son $16(38 \%)$; los operarios de almacenamiento y corte, 19 (45\%); y el personal de gestión, 7 (17\%). Existen 2 turnos de trabajo: día y noche; en el día, operan todas las áreas y, en la noche, solo opera el tratamiento del acero. En cuanto al número de trabajadores, $36(86 \%)$ laboran de día y $6(14 \%)$, de noche. Respecto de la variable condición laboral contractual, el $98 \%$ tiene contrato indefinido y solo uno es practicante.

El análisis descriptivo de las variables CLS, COS y las dimensiones de esta última: CUS y PAS, permite conocer el comportamiento de la población de estudio respecto a estas variables. La Tabla 1 muestra los estadísticos descriptivos de estas variables a nivel de toda el área de operaciones. CLS alcanza nivel intermedio (71\%); COS, nivel alto (82\%); CUS, nivel alto (85\%); y PAS, nivel intermedio (79\%).

\section{Fiabilidad de los instrumentos de medición}

Para determinar la fiabilidad de los instrumentos de medición, se evaluó la consistencia interna para
CLS, COS, CUS y PAS. Se obtuvieron los valores del coeficiente alfa de Cronbach $0.928,0.856$, 0.714 y 0.836 , respectivamente.

\section{Prueba de normalidad}

Para determinar la normalidad de los constructos, se utilizó la prueba de Kolmogorov-Smirnov, el $p$-valor obtenido fue de 0.20 tanto para CLS como para COS, lo cual indica que, para un nivel de significancia de 0.05 , ambas variables se aproximan a la normal. CUS y PAS obtuvieron $p$-valores de 0.05 y 0.04 , respectivamente. Estos resultados justifican el uso de test paramétricos para el contraste de medias, debido a que tanto el $t$ de Student como el ANOVA son, también, robustos frente a la violación del supuesto de normalidad (Sawilowsky y Blair,1992; Stonehouse y Forrester, 1998; Schmider, Ziegler, Danay, Beyer y Bühner, 2010), como en el caso de PAS, cuyo $p$-valor con tres decimales es 0.044 .

\section{Contrastación de hipótesis}

En las hipótesis 1, 2 y $3\left(\mathrm{H}_{1}, \mathrm{H}_{2}\right.$ y $\left.\mathrm{H}_{3}\right)$, las variables consideradas son ordinales, por lo que se determinó la relación existente entre las variables mediante la correlación de Spearman, la cual es apropiada para este tipo de variables, independientemente de la forma de su distribución (Warner, 2010; Ruscio, 2008). En cada caso, la hipótesis se contrastó con su correspondiente hipótesis nula $\left(\mathrm{H}_{0}\right)$ y la hipótesis alternativa $\left(\mathrm{H}_{\mathrm{a}}\right)$, donde $\mathrm{H}_{0}$ niega la relación entre las variables y $\mathrm{H}_{\mathrm{a}}$ afirma la relación. Los resultados se muestran en la Tabla 2.

Para la hipótesis $\mathrm{H}_{1}$, en el resultado de la prueba se obtiene un $p$-valor de 0.011 , que está por debajo del nivel de significancia del 0.05 , límite máximo de error ( $\alpha$ ) aceptado en el estudio. El resultado obtenido permite rechazar la $\mathrm{H}_{1-0}$ y aceptar la $\mathrm{H}_{1-\mathrm{a}}$. En consecuencia, sí existe relación entre el CLS y las COS en la presente investigación. En cuanto al coeficiente Rho de Spearman, se obtuvo 0.389, por

Tabla 1. Estadísticos de las variables CLS, COS, CUS y PAS del Área de Operaciones.

\begin{tabular}{|c|c|c|c|c|c|c|}
\hline Variable & Rango & Mínimo & Máximo & Media & Nivel & $\%$ \\
\hline CLS & 43 & 37 & 80 & 61.4 & Intermedio & 71 \\
\hline COS & 14 & 16 & 30 & 25.6 & Alto & 82 \\
\hline CUS & 6 & 9 & 15 & 13.1 & Alto & 85 \\
\hline PAS & 8 & 7 & 15 & 12.5 & Intermedio & 79 \\
\hline
\end{tabular}

N: 42 individuos.

Fuente: Elaboración propia. 
lo que existe una correlación significativa y positiva (ver Tabla 2).

Para la hipótesis $\mathrm{H}_{2}$, en el resultado de la prueba se obtiene un $p$-valor de 0.008 , menor que $\alpha=0.05$ (o $5 \%$ ). Los resultados también muestran que la correlación podría aceptarse hasta con un nivel de significancia del $1 \%$. El resultado obtenido permite rechazar la hipótesis $\mathrm{H}_{2-0}$ y aceptar la hipótesis $\mathrm{H}_{2-\mathrm{a}}$. En consecuencia, sí existe relación entre el CLS y el CUS en este estudio. El coeficiente Rho de Spearman obtenido es de 0.406 , consecuentemente, existe una correlación significativa y positiva (ver Tabla 2).

Para la hipótesis $\mathrm{H}_{3}$, en el resultado de la prueba se obtiene un $p$-valor de 0.010 , inferior al nivel de significancia del $5 \%$. Los resultados también muestran que la correlación podría aceptarse hasta con un nivel de significancia del 1\%. El resultado obtenido permite rechazar la hipótesis $\mathrm{H}_{3-0}$ y aceptar la hipótesis $\mathrm{H}_{3-\mathrm{a}}$. En consecuencia, se comprueba la relación entre el CLS y la PAS. El coeficiente Rho de Spearman obtenido es de 0.394, por lo que existe una correlación significativa y positiva (ver Tabla 2).

Mediante las hipótesis 4 y $5\left(\mathrm{H}_{4}\right.$ y $\left.\mathrm{H}_{5}\right)$ se determinó la relación entre las variables sociolaborales (consideradas como variables independientes o factores) con el CLS y las COS (consideradas como variables dependientes). Se utilizó la prueba $t$ para muestras independientes o ANOVA de un factor, según correspondió. Se contrastó la hipótesis nula $\left(\mathrm{H}_{0}\right)$ con la hipótesis alternativa $\left(\mathrm{H}_{\mathrm{a}}\right)$, donde $\mathrm{H}_{0}$ niega la influencia de la variable sociolaboral respecto a las variables de estudio (CLS, COS) y $\mathrm{H}_{\mathrm{a}}$ afirma tal influencia.

\section{Influencia de la edad en CLS, COS, CUS y PAS}

La prueba de homogeneidad de varianzas con el estadístico de Levene a un nivel de significancia del $5 \%$ dio $p$-valores de $0.251,0.013,0.944$ y 0.047 para CLS, COS, CUS y PAS, respectivamente, lo cual indica que las varianzas son homogéneas para CLS y CUS, y no para COS y PAS, por lo que se aplicó el estadístico de Welch (ver Tabla 3), como alternativa al estadístico $\mathrm{F}$ del ANOVA clásico, por ser más apropiado cuando las varianzas no son homogéneas (International Business Machines Corporation, 2019). Respecto a la prueba ANOVA, se obtuvo $p$-valores para CLS, COS, CUS y PAS de $0.835,0.294,0.944$ y 0.047 , respectivamente (ver Tabla 3); en los casos de CLS, COS y CUS, el $p$-valor es mayor que 0.05. Consecuentemente, se acepta $\mathrm{H}_{0}$, es decir, que estadísticamente la variable independiente edad no influye en CLS, COS y CUS.

Tabla 2. Correlaciones de Spearman.

\begin{tabular}{|l|l|c|c|c|c|}
\hline \multicolumn{2}{|c|}{ Variable } & CLS & COS & CUS & PAS \\
\hline \multirow{2}{*}{ CLS } & Coeficiente de correlación & 1.000 & $0.389^{*}$ & $0.406^{* *}$ & $0.394^{* *}$ \\
\cline { 2 - 6 } & Sig. (bilateral) & & 0.011 & 0.008 & 0.010 \\
\hline \multirow{2}{*}{$\cos$} & Coeficiente de correlación & $0.389^{*}$ & 1.000 & $0.865^{* *}$ & $0.940^{* *}$ \\
\cline { 2 - 6 } & Sig. (bilateral) & 0.011 & & 0.000 & 0.000 \\
\hline
\end{tabular}

* La correlación es significativa en el nivel 0.05 (bilateral).

** La correlación es significativa en el nivel 0.01 (bilateral).

$\mathrm{N}: 42$ individuos.

Fuente: Elaboración propia.

Tabla 3. Influencia de la edad en CLS, COS, CUS y PAS.

\begin{tabular}{|c|c|c|c|c|c|c|}
\hline \multirow[b]{2}{*}{ Variables } & \multicolumn{2}{|c|}{ Prueba de igualdad de varianzas } & \multicolumn{2}{|c|}{ Prueba robusta de Welch } & \multicolumn{2}{|c|}{ ANOVA } \\
\hline & Estadístico de Levene & Sig. & Estadístico $^{\mathrm{a}}$ & Sig. & $\mathbf{F}$ & Sig. \\
\hline CLS & 1.431 & 0.251 & 0.231 & 0.795 & 0.181 & 0.835 \\
\hline $\cos$ & 4.891 & 0.013 & 0.994 & 0.386 & 1.265 & 0.294 \\
\hline CUS & 2.221 & 0.944 & 0.078 & 0.925 & 0.058 & 0.944 \\
\hline PAS & 2.221 & 0.047 & 2.402 & 0.114 & 3.322 & 0.047 \\
\hline
\end{tabular}

a $\mathrm{F}$ distribuida de forma asintótica.

Fuente: Elaboración propia. 
La prueba robusta de Welch, que procede para la relación edad y COS, confirma el hallazgo obtenido en el ANOVA. En el caso de PAS, el $p$-valor obtenido en el ANOVA es menor que 0.05. De este modo, la edad sí influye en PAS, aunque este resultado se debe asumir con precaución, pues está muy cercano al valor de significancia mínimo aceptado (5\%) $y$, para el caso de la prueba robusta de Welch, no resultó significativo.

\section{Influencia del grado de instrucción en CLS, COS, CUS y PAS}

La prueba de homogeneidad de varianzas con el estadístico de Levene a un nivel de significancia del $5 \%$ dio $p$-valores de $0.503,0.862,0.943$ y 0.672 para CLS, COS, CUS y PAS, respectivamente, lo cual indica que las varianzas son homogéneas para todas las variables con respecto a la variable grado de instrucción (ver Tabla 4). Respecto a las pruebas de ANOVA, los $p$-valores obtenidos son de 0.357 , $0.585,0.415$ y 0.563 para CLS, COS, CUS y PAS, respectivamente (ver Tabla 4); en todos los casos, el $p$-valor es mayor que 0.05. Consecuentemente, se acepta $\mathrm{H}_{0}$, es decir, que estadísticamente la variable independiente grado de instrucción no influye en CLS, COS, CUS y PAS.

\section{Influencia del turno laboral en CLS, COS, CUS y PAS}

La prueba de homogeneidad de varianzas con el estadístico de Levene a un nivel de significancia del $5 \%$ dio $p$-valores de $0.550,0.066,0.006$ y 0.171 para CLS, COS, CUS y PAS, respectivamente, lo cual indica que las varianzas son homogéneas para todas las variables con respecto a la variable turno laboral (ver Tabla 5), salvo para CUS. Respecto a las pruebas $t$ para muestras independientes, se obtuvieron $p$-valores de $0.585,0.693,0.394$ y 1.000 para CLS, COS, CUS y PAS, respectivamente (ver Tabla 5); en todos los casos, el $p$-valor es mayor que 0.05 . En consecuencia, se acepta $\mathrm{H}_{0}$, es decir, que estadísticamente la variable independiente turno laboral no influye en CLS, COS, CUS y PAS.

\section{Influencia del puesto laboral en CLS, COS, CUS y PAS}

La prueba de homogeneidad de varianzas con el estadístico de Levene a un nivel de significancia del $5 \%$ dio $p$-valores de $0.878,0.345,0.176$ y 0.390 para CLS, COS, CUS y PAS, respectivamente, lo cual indica que las varianzas son homogéneas para todas las variables en relación a la variable puesto laboral (ver Tabla 6). Respecto a la prueba ANOVA, se obtuvieron $p$-valores de $0.107,0.218,0.389$ y 0.191 para CLS, COS, CUS y PAS respectivamente (ver Tabla 6); en todos los casos el $p$-valor es mayor que 0.05. De este modo, se acepta $\mathrm{H}_{0}$, es decir, que estadísticamente la variable independiente puesto laboral no influye en CLS, COS, CUS y PAS.

\section{Influencia del tiempo de servicio en CLS, COS, CUS y PAS}

La prueba de homogeneidad de varianzas con el estadístico de Levene a un nivel de significancia

Tabla 4. Influencia de grado de instrucción en CLS, COS, CUS y PAS.

\begin{tabular}{|c|c|c|c|c|}
\hline \multirow{2}{*}{ Variables } & \multicolumn{2}{|c|}{ Prueba de igualdad de varianzas } & \multicolumn{2}{c|}{ ANOVA } \\
\cline { 2 - 5 } & Estadístico de Levene & Sig. & 1.059 & 0.357 \\
\hline CLS & 0.699 & 0.503 & 0.544 & 0.585 \\
\hline COS & 0.150 & 0.862 & 0.899 & 0.415 \\
\hline CUS & 0.059 & 0.943 & 0.583 & 0.563 \\
\hline PAS & 0.401 & 0.672 & & \\
\hline
\end{tabular}

Fuente: Elaboración propia.

Tabla 5. Influencia del turno laboral en CLS, COS, CUS y PAS

\begin{tabular}{|c|c|c|c|c|}
\hline \multirow{2}{*}{ Variables } & \multicolumn{2}{|c|}{ Prueba de igualdad de varianzas } & \multicolumn{2}{c|}{ Prueba t } \\
\cline { 2 - 5 } & Estadístico de Levene & Sig. & 0.550 & 0.585 \\
\hline CLS & 0.364 & 0.550 & 0.397 & 0.693 \\
\hline COS & 3.570 & 0.066 & 0.861 & 0.394 \\
\hline CUS & 8.484 & 0.006 & 0.000 & 1.000 \\
\hline PAS & 1.943 & 0.171 & & \\
\hline
\end{tabular}

Fuente: Elaboración propia. 
del $5 \%$ dio $p$-valores de $0.064,0.380,0.308$ y 0.105 para CLS, COS, CUS y PAS, respectivamente, lo cual indica que las varianzas son homogéneas para todas las variables en relación a la variable tiempo de servicio (ver Tabla 7). Respecto a la prueba ANOVA se obtuvo un $p$-valor de 0.956, 0.164, 0.856 y 0.018 para CLS, COS, CUS y PAS, respectivamente (ver Tabla 7); en los casos de CLS, COS y CUS el $p$-valor es mayor que 0.05; consecuentemente se acepta $\mathrm{H}_{0}$, es decir, que estadísticamente la variable independiente tiempo de servicio no influye en CLS, COS y CUS. En el caso de PAS, el $p$-valor es menor que 0.05; por lo tanto, el tiempo de servicio sí influye en PAS.

\section{DISCUSIÓN}

La fiabilidad de los instrumentos de medición fue muy similar a lo reportado por otros autores. Para el clima de seguridad laboral (CLS), se encontró un alfa de Cronbach de 0.93 , el cual es muy cercano al valor de 0.95 hallado por Zohar y Luria (2005) y superior al 0.77 reportado por Zhou y Jiang (2015). En cuanto a las conductas de seguridad (COS), se obtuvo 0.86; en el cumplimiento de la seguridad (CUS) y la participación en la seguridad (PAS), 0.71 y 0.86 , respectivamente; en comparación a trabajos previos, como el de Neal y Griffin (2006), los valores encontrados fueron de 0.93 y 0.89 , respectivamente, con una gran cercanía a la variable PAS.

Mediante la hipótesis $\mathrm{H}_{1}$ se demostró que existe relación significativa y positiva entre el CLS y las
COS, tal como se evidenció en estudios anteriores (Manning, Davidson y Manning, 2005; Lyu, Hon, Chan, Wong y Javed, 2018; Abubakar, Karadal, Bayighomog y Merdan, 2018). Adicionalmente, con las hipótesis $\mathrm{H}_{2}$ y $\mathrm{H}_{3}$ también se demostró que existe relación significativa y positiva entre el clima de seguridad laboral y el cumplimiento de la seguridad (CUS) y la participación en la seguridad (PAS), lo cual ha sido probado en una empresa de la industria del acero en el Perú y confirma lo planteado por diferentes autores (Hon, Chan y Yam, 2014; Zohar y Luria, 2005; Neal y Griffin, 2006; Lyu et al., 2018; Dahl y Kongsvik, 2018; Mirza, Isha, Memon, Azeem y Zahid, 2019).

En consecuencia, el clima de seguridad laboral (CLS) está relacionado con las conductas de seguridad (COS) de los trabajadores. En esta investigación, se encontró correlación significativa y positiva de CLS con CUS y PAS, inclusive al nivel de significancia de 0.01 , es decir, $99 \%$ de confianza de que la correlación sea verdadera. Con respecto a CLS con COS, la correlación se da al nivel de significancia de 0.05 .

A través de la prueba ANOVA de un factor, se encontró que solo la edad y el tiempo de servicio de los trabajadores tienen influencia sobre su participación en la seguridad, lo cual apoya parcialmente los hallazgos previos que demuestran la incidencia de las variables sociolaborales en el CLS y las COS (Fang, Chen y Wong, 2006; Xiong, Liang, Luo y Fung, 2018; Wang, Mei, Liu, Zhou y Zhang, 2019).

Tabla 6. Influencia del puesto laboral en CLS, COS, CUS y PAS.

\begin{tabular}{|c|c|c|c|c|}
\hline \multirow{2}{*}{ Variables } & \multicolumn{2}{|c|}{ Prueba de igualdad de varianzas } & \multicolumn{2}{c|}{ ANOVA } \\
\cline { 2 - 5 } & Estadístico de Levene & Sig. & 2.372 & 0.107 \\
\hline CLS & 0.131 & 0.878 & 1.586 & 0.218 \\
\hline COS & 1.095 & 0.345 & 0.967 & 0.389 \\
\hline CUS & 1.818 & 0.176 & 1.728 & 0.191 \\
\hline PAS & 0.965 & 0.390 & & F \\
\hline
\end{tabular}

Fuente: Elaboración propia.

Tabla 7. Influencia del tiempo de servicio en CLS, COS, CUS y PAS.

\begin{tabular}{|c|c|c|c|c|}
\hline \multirow{2}{*}{ Variables } & \multicolumn{2}{|c|}{ Prueba de igualdad de varianzas } & \multicolumn{2}{c|}{ ANOVA } \\
\cline { 2 - 5 } & Estadístico de Levene & Sig. & 0.045 & 0.956 \\
\hline CLS & 2.947 & 0.064 & 1.897 & 0.164 \\
\hline COS & 0.992 & 0.380 & 0.156 & 0.856 \\
\hline CUS & 1.214 & 0.308 & 4.466 & 0.018 \\
\hline PAS & 2.385 & 0.105 & & F \\
\hline
\end{tabular}

Fuente: Elaboración propia. 


\section{CONCLUSIONES}

1. El clima de seguridad laboral (CLS) se relaciona positiva y significativamente con las conductas de seguridad (COS) en una muestra de trabajadores de una empresa de la industria del acero ubicada en Perú.

2. El clima de seguridad laboral (CLS) se relaciona directa y positivamente en la variable cumplimiento de la seguridad (CUS). Una mejora en CLS implica una mejora en CUS y viceversa.

3. El clima de seguridad laboral (CLS) se relaciona directa y positivamente en la variable participación en la seguridad (PAS). Una mejora en CLS implica una mejora en PAS y viceversa.

4. No se encontraron relaciones de causalidad de las variables sociolaborales con el CLS y las COS respecto a los trabajadores del área de operaciones de la presente investigación. No obstante, los resultados muestran comportamientos o tendencias que podrían confirmar la causalidad con una muestra más grande.

5. Se encontró una relación de causalidad de las variables sociolaborales edad y tiempo de servicio de los trabajadores en la participación en la seguridad (PAS).

\section{REFERENCIAS BIBLIOGRÁFICAS}

[1] Abubakar, A. M., Karadal, H., Bayighomog, S. W. y Merdan, E. (9 de mayo de 2018). Workplace Injuries, Safety Climate and Behaviors: Application of an Artificial Neural Network. International Journal of Occupational Safety and Ergonomics. Recuperado de https:// www.tandfonline.com/doi/full/10.1080/1080354 8.2018.1454635.

[2] Coyle, I. R., Sleeman, S. D. y Adams, N. (1995). Safety Climate. Journal of Safety Research, 26(4), 247-254.

[3] Dahl, Ø. y Kongsvik, T. (2018). Safety Climate and Mindful Safety Practices in the Oil and Gas Industry. Journal of Safety Research, 64, 29-36.

[4] Díaz, A. (2006). Auditoría del clima y cultura de seguridad en la empresa. (Tesis doctoral). Universidad de Valencia, España. Recuperado de https://www.tdx.cat/bitstream/ handle/10803/10188/diaz.pdf.

[5] Fang, D., Chen, Y. y Wong, L. (2006). Safety Climate in Construction Industry: A Case Study in
Hong Kong. Journal of Construction Engineering and Management, 132(6), 573-584.

[6] Griffin, M. A. y Neal, A. (2000). Perceptions of Safety at Work: A Framework for Linking Safety Climate to Safety Performance, Knowledge, and Motivation. Journal of Occupational Health Psychology, 5(3), 347-358.

[7] Hon, C. K. H., Chan, A. P. C. y Yam, M. C. H. (2014). Relationships Between Safety Climate and Safety Performance of Building Repair, Maintenance, Minor Alteration, and Addition (RMAA) Works. Safety Science, 65, 10-19.

[8] International Business Machines Corporation (IBM) (2019). SPSS Statistics 25.0.0. ANOVA de un factor: Opciones. España: IBM Knowledge Center. Recuperado de https:// www.ibm.com/support/knowledgecenter/es/ SSLVMB 25.0.0/statistics mainhelp_ddita/ spss/base/idh_onew_opt.html.

[9] Johnson, S. E. (2007). The Predictive Validity of Safety Climate. Journal of Safety Research, 38(5), 511-521.

[10] Lyu, S., Hon, C. K. H., Chan, A. P. C., Wong, F. K. W. y Javed, A. A. (2018). Relationships among Safety Climate, Safety Behavior, and Safety Outcomes for Ethnic Minority Construction Workers. International Journal of Environmental Research and Public Health, 15(484), 1-16.

[11] Manning, M. L., Davidson, M. y Manning, R. L. (2005). Measuring Tourism and Hospitality Employee Workplace Perceptions. International Journal of Hospitality Management, 24(1), 7590.

[12] Melià, J. L. y Sesé, A. (1999). La medida del clima de seguridad y salud laboral. Anales de Psicología, 15(2), 269-289.

[13] Mirza, M., Isha, A., Memon, M., Azeem, S. y Zahid, M. (15 de mayo de 2019). Psychosocial Safety Climate, Safety Compliance and Safety Participation: The Mediating Role of Psychological Distress. Journal of Management \& Organization. Recuperado de https://www.cambridge.org/core/journals/ journal-of-management-and-organization/ article/psychosocial-safety-climate-safetycompliance-and-safety-participation-themediating-role-of-psychological-distress/942B D88321E73482B4A4D99A3D2D0D9A.

[14] Neal, A. y Griffin, M. A. (2004). Safety Climate and Safety at Work. En J. Barling y M. R. Frone (Eds.), The Psychology of Workplace Safety 
(pp. 15-34). Washington D. C., Estados Unidos: American Psychological Association.

[15] Neal, A. y Griffin, M. A. (2006). A Study of the Lagged Relationships Among Safety Climate, Safety Motivation, Safety Behaviour and Accidents at the Individual and Groups Levels. Journal of Applied Psychology, 91(4), 946-953.

[16] Peiró, J. M. (1984). Psicología de la organización. Madrid, España: Universidad Nacional de Educación a Distancia (UNED).

[17] Ruscio, J. (2008). Constructing Confidence Intervals for Spearman's Rank Correlation with Ordinal Data: A Simulation Study Comparing Analytic and Bootstrap Methods. Journal of Modern Applied Statistical Methods, 7(2), 416434.

[18] Sawilowsky, S. y Blair, R. C. (1992). A More Realistic Look at the Robustness and Type II Error Probabilities of the Test to Departures from Population Normality. Psychological Bulletin, 111(2), 352-360.

[19] Schmider, E., Ziegler, M., Danay, E., Beyer, L. y Bühner, M. (2010). Is It Really Robust? Reinvestigating the Robustness of ANOVA Against Violations of the Normal Distribution Assumption. Methodology: European Journal of Research Methods for the Behavioral and Social Sciences, 6(4), 147-151.

[20] Stonehouse, J. M. y Forrester, G. J. (1998). Robustness of the $t$ and $U$ tests under combined assumption violations. Journal of Applied Statistics, 25(1), 63-74.
[21] Wang, Q., Mei, Q., Liu, S., Zhou, Q. y Zhang, J. (2019). Demographic Differences in Safety Proactivity Behaviors and Safety Management in Chinese Small-Scale Enterprises. Safety Science, 120, 179-184.

[22] Warner, P. (2010). Quantifying Association in Ordinal Data. Journal of Family Planning and Reproductive Health Care, 36(2), 83-85.

[23] Xiong, C., Liang, K., Luo, H. y Fung, I. W. H. (2018). Identification of Safety-Related Opinion Leaders among Construction Workers: Evidence from Scaffolders of Metro Construction in Wuhan, China. International Journal of Environmental Research and Public Health, 15(2176), 1-17.

[24] Zhou, F. y Jiang, C. (2015). Leader-Member Exchange and Employees' Safety Behavior: The Moderating Effect of Safety Climate. Procedia Manufacturing, 3, 5014-5021.

[25] Zohar, D. (1980). Safety Climate in Industrial Organizations: Theoretical and Applied Implications. Journal of Applied Psychology, 65(1), 96-102.

[26] Zohar, D. y Luria, G. (2005). A Multilevel Model of Safety Climate: Cross-Level Relationships Between Organization and Group-Level Climates. Journal of Applied Psychology, 90(4), 616-628. 


\section{Safety Climate and Safety Behavior in a Steel Industry Company in Peru}

Manuel Enrique Herrera Díaz ${ }^{1}$

\begin{abstract}
Workers are the main axis of occupational safety and health, which is why companies should direct efforts towards learning more about their human capital, raise their awareness and ensure their participation in preventive safety management. The variables used in this research are occupational safety climate (OSC), safety behaviors (SB) and socio-occupational variables. Reliability was verified using Cronbach's alpha and the relationships between variables were evaluated using Spearman's correlation coefficient, t-tests and ANOVA. The main results are 1) the reliability of the constructs, 2) verification of significant and positive correlation between OSC and SB, 3) no causal relationships were found between the socio-occupational variables and OSC and $\mathrm{SB}$, and 4) socio-occupational variables age and time of service influence the variable safety participation (SP) in the workplace.
\end{abstract}

Keywords: safety climate; safety behaviors; safety compliance; safety participation.

\section{INTRODUCTION}

Prevention is the foundation of occupational safety and health $(\mathrm{OSH})$, from which several models and management systems have been developed, strengthened by national laws and international conventions; however, over time it has become clear that they have not sufficed to counteract accidents at work and to optimize safety in organizations. Preventive systems in companies are most effective when they seek the participation and awareness of their workers, in order to develop a preventive safety culture. On the other hand, safety often competes with other organizational interests, such as quality and productivity, which can lead to a weakening of workers' safe behavior, a decline in prevention culture, and give rise to accidents at work, among other consequences. In this sense, it is vital to monitor working environment and workers' behavior, in order to detect risks early and turn them into opportunities for improvement. This study considers two variables that aim to improve and strengthen occupational safety management, which are occupational safety climate and workers' safety behavior.

The complexity and magnitude of organizational climate merits greater specificity for specific purposes and, thus, for measures designed for specific working climates. The characteristic of organizational climate of acting as a precedent of workers' behavior turns safety climate into an important tool with respect to the behaviors of workers in occupational safety and health. In 1980, Dov Zohar coined the concept of security climate; he noted that organizational factors affect safety-related outcomes, and emphasized the importance of considering specific climates as an organization's safety climate, for which he drew a distinction between global measures and particular measures. Thus, safety climate is defined as the perceptions that workers share about the aspects related to safety at work, with emphasis on the attitudes and behaviors of supervisors and managers (Zohar, 1980). Safety climate assesses workers' shared perceptions of policies

1 Electronic engineer, specializing in Business Management, Information Technology and Communications. Currently working as independent consultant in Business Management, Operations Management, Supply Chain, Safety and Occupational Health. (Lima, Peru). ORCID: https://orcid.org/0000-0002-4759-9554

E-mail: menriqueherrera@gmail.com 
(strategic level), procedures (tactical level) and safety practices (operational level) in the organization and understanding of the impact that the company has on the safety behavior or performance of its workers (Díaz, 2006).

Zohar and Luria (2005) developed the multilevel safety climate (MSC) model, which focuses on management policies, procedures and practices as primary sources or references for climate safety perceptions. They proposed a global safety climate at organization level and a more specific climate at group level, where policies and procedures provide references at organization level, while its daily implementation by front-line supervisors provides referrals at group level. These authors created an instrument to measure safety climate at group level by means of a 16-item questionnaire, with a $\mathrm{Li}$ kert-5 response format, with which they obtained a very high reliability with a Cronbach's alpha of 0.95 . Likewise, they also determined strong correlations between factor loadings greater than 0.80 using a single factor for this safety climate and the validity of the construct.

Johnson (2007) conducted a study to verify the predictive character of "safety climate" with respect to safety results (behavior, accidents, etc.). For that purpose, he applied the MSC model and the safety climate questionnaire of Zohar and Luria (2005) to 292 workers in 20 manufacturing companies. Using a model of structural equations, he found a strong correlation between factors, greater than 0.95, that demonstrated and reinforced Zohar and Luria's proposal, indicating that the single-factor structure provides an appropriate data fit and represents the commitment or priority of management towards safety. The author concludes that a) the multi-level safety model (MSC) questionnaire is psychometrically reliable and valid, and b) serves as an effective predictor of safety outcomes (behavior and accidents).

Along these lines, Neal and Griffin (2004) argued that the relationship between safety climate and accidents at work is mediated by safety behavior. In other words, safety behavior is the stronger predictor of accidents in the workplace.

Occupational safety climate is conceptualized as: a) workers' subjective perception regarding the organization, its members, structure, and processes; that b) despite individual differences, it has common perceptions; c) it is based on objective elements of the environment or indicators, and that, in addition, d) it behaves as a precursor of the workers' behavior, a quality that gives it its real significance (Peiró,
1984; Coyle, Sleeman \& Adams, 1995; Melià \& Sesé, 1999).

Based on the theory of job performance in the field of occupational safety, the two-dimensional model of workers' safety behavior was developed. Its main promoters are Griffin and Neal (2000) and their model was based on two dimensions: safety compliance and participation. Safety compliance involves the tasks that workers must inevitably perform in order to maintain workplace safety. These activities are carried out in accordance to standards and procedures for the preservation of OSH. Safety participation describes behaviors that indirectly contribute to individual and collective safety within the organization, which help to develop a safe environment, that is, support safety in the broad organizational context. These behaviors are oriented to voluntary safety behaviors of workers.

In this research, safety behaviors (dependent variable in this study) and its two dimensions, safety compliance and safety participation were analyzed based on Neal and Griffin's scale (2006), through six statements. In their original study, these authors obtained a reliability $\alpha=0.93$ for the safety compliance dimension and $\alpha=0.89$ for the safety participation dimension. The two dimensions were highly correlated at group level; therefore, a single indicator for safety behaviors is formulated and its construct validity is confirmed $(r=0.91, p=0.001)$.

This study was conducted at a steel industry company in Peru. The following acronyms are used hereafter: ocupational safety climate (OSC), safety behaviors (SB), safety compliance (SC) and safety participation (SP).

\section{Hypotheses and variables}

$\mathrm{H}_{1}$ : There is a relationship between occupational safety climate (OSC) and safety behaviors (SB) of workers in the operations area of a steel industry company in Peru.

$\mathrm{H}_{2}$ : There is a relationship between occupational safety climate (OSC) and safety compliance (SC) of workers in the operations area of a steel industry company in Peru.

$\mathrm{H}_{3}$ : There is a relationship between occupational safety climate (OCS) and safety participation (SP) of workers in the operations area of a steel industry company in Peru.

$\mathrm{H}_{4}$ : There is a causal relationship between the socio-occupational variables and occupational safety 
climate (OSC) of workers in the operations area of a steel industry company in Peru.

$\mathrm{H}_{5}$ : There is a causal relationship between the socio-occupational variables and safety behaviors (SB) of workers in the operations area of a steel industry company in Peru

Additionally, to establish causal relationship, socio-occupational variables were considered as independent variables in $\mathrm{H}_{4}$ and $\mathrm{H}_{5}$, OSC was considered a dependent variable in $\mathrm{H}_{4}$, and so were $\mathrm{SB}$, $\mathrm{SC}$ and $\mathrm{SP}$ in $\mathrm{H}_{5}$.

\section{METHODOLOGY}

This is a quantitative research project, because it measures the variables safety climate and safety behaviors according to their magnitude, data are then examined numerically using statistical tools following a non-experimental design. The method used is cross-sectional-descriptive and correlational. It is cross-sectional because data will be collected in a single moment and time; and it is descriptive and correlational because the purpose is to describe both the OSC and the SB, and to analyze their interrelation. It is causal when socio-occupational variables are compared against OSC, SB, SC and SP.

\section{Participants}

The study focuses on workers in the operations area of a steel industry company in Peru. Two main activities are conducted in this area: a) heat treatment of steel and b) storage and cutting of steel materials. Heat treatment is applied to steel parts with the aim of improving their mechanical properties, especially hardness, strength and elasticity, by applying different processes such as tempering, hardening, cementation, nitriding, annealing, etc., wherein high temperatures are used. In storage and cutting operations, operators mainly handle and cut very heavy and large steel bars and plates. This study was conducted on the total number of workers in the operations area, including management staff, that is, on 42 workers.

\section{Measuring instrument}

Occupational safety climate was measured with the multilevel safety model designed by Zohar and Luria (2005), consisting of 16 items; SB was measured with the scale proposed by Neal and Griffin (2006), consisting of six statements, where the first three measure SC dimension and the remaining three measure SP dimension. Likert 5-point scale questionnaires were applied, the options were
1 = completely disagree, 2 = disagree, $3=$ neither agree nor disagree, $4=$ agree and $5=$ completely agree.

Occupational safety climate, SB, SC and SP are qualitative-ordinal variables and their value ranges are as follows: OSC, from 16 to 80 ; SB, from 6 to 30 ; SC and SP, from 3 to 15 . For a better understanding, the values obtained were expressed in percentages, from the maximum to minimum value that each variable could take, and in levels, proposed by the author of this study, using the following ranges: poor $(\mathrm{P}), 50 \%$; very low $(\mathrm{VL})$, from $51 \%$ to $60 \%$; low (L), from $61 \%$ to $70 \%$; intermediate (I), from $71 \%$ to $80 \%$; high $(\mathrm{H})$, from $81 \%$ to $90 \%$; and very high (VH), from $91 \%$ to $100 \%$.

\section{Procedure}

Measuring instruments were applied over a twoweek period. The process was supported by the general management and the participation of the human resources and occupational safety departments. All questionnaire responses were anonymous, all previously coordinated with the areas indicated above and supervised by a specialist. On average, the assessment lasted 30 minutes. The data obtained were then processed using the statistical software SPSS (version 25).

\section{RESULTS}

\section{Descriptive analysis}

Through the analysis of socio-occupational variables, a greater knowledge of the study population was achieved. The socio-occupational variables examined are gender, age, level of education, shift, job position, contractual status and time of service in the company (experience). Out of the total number of workers, 95 per cent are men and 5 per cent are women. Age ranges from 22 to 64 , with an average age of 38 ; workers were grouped into three categories in this study ( $\leq 30$, from 31 to 45 and $\geq$ 46 years old). The time of service (in years) has an average of 10 and a range of 1 to 47 years, and it was also grouped into three categories $(\leq 1$, from 2 to 14 and $\geq 15$ ). As for the level of education, the distribution is: elementary $12 \%$, secondary $57 \%$, and higher $31 \%$. There are $16(38 \%)$ steel treatment operators, $19(45 \%)$ storage and cutting operators, and $7(17 \%)$ management staff members. There are two shifts: day and night; during the day, all areas operate and, at night, only steel treatment. In terms of the number of workers, 36 (86\%) work during the day and $6(14 \%)$ at night. Regarding the variable 
contractual status, $98 \%$ have a permanent contract and only one is an intern.

The descriptive analysis of variables OSC, SB and the dimensions of the latter, SC and SP, reveals the behavior of the study population with respect to these variables. Table 1 shows the descriptive statistics of these variables at the entire operations area level. OSC reaches intermediate level (71\%); SB, high level (82\%); SC, high level (85\%); and SP, intermediate level (79\%).

\section{Reliability of measuring instruments}

Internal consistency of OSC, SB, SC, and SP was assessed to determine the reliability of the measuring instruments. Cronbach's alpha coefficients equal to $0.928,0.856,0.714$, and 0.836 were obtained, respectively.

\section{Normality test}

The Kolmogorov-Smirnov test was used to determine the normality of the constructs, and a $p$-value of 0.20 was obtained for OSC and SB, which indicates that, considering significant level at 0.05 , both variables approximate to a normal distribution. Safety compliance and SP obtained $p$-values of 0.05 and 0.04 , respectively. These results justify the use of parametric tests for the contrast of means, because both the Student's $t$ and ANOVA tests are robust against the violation of the normality assumption (Sawilowsky \& Blair, 1992; Stonehouse \& Forrester, 1998; Schmider, Ziegler, Danay, Beyer \& Bühner, 2010 ), as is the case of SP, with a $p$-value of 0.044 .

\section{Hypothesis testing}

In hypotheses 1,2 and $3\left(\mathrm{H}_{1}, \mathrm{H}_{2}\right.$ and $\left.\mathrm{H}_{3}\right)$, variables are ordinal, thus the relationship between them was determined using Spearman's correlation, which is appropriate for this type of variables, regardless of their distribution (Warner, 2010; Ruscio, 2008). In each case, the hypothesis was contrasted with its corresponding null hypothesis $\left(\mathrm{H}_{0}\right)$ and the alternative hypothesis $\left(\mathrm{H}_{\mathrm{a}}\right)$, where $\mathrm{H}_{0}$ denies the relationship between the variables and $\mathrm{H}_{\mathrm{a}}$ asserts the relationship. Results are shown in Table 2.

A $p$-value of 0.011 was obtained for $H_{1}$, which is below the significance level of 0.05 , the maximum margin of error $(\alpha)$ accepted in the study. The result rejects $\mathrm{H}_{1-0}$ and accepts $\mathrm{H}_{1-\mathrm{a}}$. Consequently, there is a relationship between OSC and SB in this study. $A$ Spearman's Rho coefficient of 0.389 was obtained, therefore, there is a significant and positive correlation between both variables (see Table 2).

A $p$-value of 0.008 was obtained for $\mathrm{H}_{2}$, less than $\alpha$ $=0.05$ (or 5\%). Results also show that the correlation could be accepted up to a significance level of $1 \%$. The result rejects $\mathrm{H}_{2-0}$ and accepts $\mathrm{H}_{2-\mathrm{a}}$. Consequently, there is a relationship between OSC and $\mathrm{SC}$ in this study. A Spearman's Rho coefficient of 0.406 was obtained, therefore, there is a significant and positive correlation between both variables (see Table 2).

A p-value of 0.010 was obtained for $\mathrm{H}_{3}$, which is below the significance level of $5 \%$. Results also show that the correlation could be accepted up to a significance level of $1 \%$. The result rejects $\mathrm{H}_{3-0}$ and accepts $\mathrm{H}_{3-\mathrm{a}}$. Consequently, the relationship between OSC and SP in this study is verified. A Spearman's Rho coefficient of 0.394 was obtained, therefore, there is a significant and positive correlation between both variables (see Table 2).

Hypotheses 4 and $5\left(\mathrm{H}_{4}\right.$ and $\left.\mathrm{H}_{5}\right)$ determined the relationship between socio-occupational variables (independent variables or factors) and OSC and SB (dependent variables). The $t$-test or one-way ANOVA were used for independent samples, as appropriate. The null hypothesis $\left(\mathrm{H}_{0}\right)$ was contrasted against the alternative hypothesis $\left(\mathrm{H}_{\mathrm{a}}\right)$, where $\mathrm{H}_{0}$ denies the influence of the socio-occupational variable with respect to the other variables (OSC, $\mathrm{SB}$ ) and $\mathrm{H}_{\mathrm{a}}$ asserts such influence.

Table 1. Statistics of variables OSC, SB, SC and SP in the operations area.

\begin{tabular}{|c|c|c|c|c|c|c|}
\hline Variable & Range & Minimum & Maximum & Mean & Level & $\%$ \\
\hline OSC & 43 & 37 & 80 & 61.4 & Intermediate & 71 \\
\hline SB & 14 & 16 & 30 & 25.6 & High & 82 \\
\hline SC & 6 & 9 & 15 & 13.1 & High & 85 \\
\hline SP & 8 & 7 & 15 & 12.5 & Intermediate & 79 \\
\hline
\end{tabular}

$\mathrm{N}$ : 42 individuals.

Source: Prepared by the author. 


\section{Influence of age on OSC, SB, SC, and SP}

The homogeneity test of variances and Levene's test at a significance level of $5 \%$ yielded $p$-values of $0.251,0.013,0.944$ and 0.047 for OSC, SB, SC and SP, respectively, indicating homogeneity of variances for OSC and SC, but not for SB and SP. Therefore, Welch's test was applied (see Table 3), as an alternative to the classical ANOVA F-statistic, as it is more appropriate when variances are not homogeneous (International Business Machines Corporation, 2019). P-values of 0.835, 0.294, 0.944 and 0.047 were obtained for OSC, SB, SC and SP, respectively (see Table 3), using ANOVA; a $p$-value greater than 0.05 was obtained for OSC, SB and SC. Consequently, $\mathrm{H}_{0}$ is accepted, that is, statistically, independent variable age does not influence OSC, SB nor SC.

Welch's test, used to analyze the relationship between age and SB, corroborates the results obtained in the ANOVA. The $p$-value obtained for SP in the ANOVA is less than 0.05; therefore, age does influence SP. This result should, however, be taken carefully, as it is very close to the minimum accepted significance level $(5 \%)$ and it was not significant in the Welch's test.
Influence of level of education on OSC, SB, SC, and SP

The homogeneity test of variances and the Levene's test at a significance level of $5 \%$ yielded $p$-values of $0.503,0.862,0.943$ and 0.672 for OSC, SB, SC and SP, respectively, indicating homogeneity of variances for all variables with respect to level of education (see Table 4). $P$-values of $0.357,0.585$, 0.415 and 0.563 were obtained for OSC, SB, SC and SP, respectively (see Table 4), using ANOVA; a $p$-value greater than 0.05 was obtained for all cases. Consequently, $\mathrm{H}_{0}$ is accepted, that is, statistically, independent variable level of education does not influence OSC, SB, SC nor SP.

\section{Influence of work shift on OSC, SB, SC and SP}

The homogeneity test of variances and Levene's test at a significance level of $5 \%$ yielded $p$-values of $0.550,0.066,0.006$ and 0.171 for OSC, SB, SC and $\mathrm{SP}$, respectively, indicating homogeneity of variances for all variables with respect to variable work shift (see Table 5), except for SC. Regarding the $t$-tests for independent samples, $p$-values of $0.585,0.693$, 0.394 and 1.000 were obtained for OSC, SB, SC and $\mathrm{SP}$, respectively (see Table 5); a $p$-value greater than 0.05 was obtained for all cases. Consequently, $\mathrm{H}_{0}$ is

Table 2. Spearman's correlation.

\begin{tabular}{|l|l|c|c|c|c|}
\hline \multicolumn{2}{|l|}{ Variable } & OSC & SB & SC & SP \\
\hline \multirow{2}{*}{ OSC } & Correlation coefficient & 1.000 & $0.389^{*}$ & $0.406^{* *}$ & $0.394^{* *}$ \\
\cline { 2 - 6 } & Sig. (2-tailed) & & 0.011 & 0.008 & 0.010 \\
\hline \multirow{2}{*}{ SB } & Correlation coefficient & $0.389^{*}$ & 1.000 & $0.865^{* *}$ & $0.940^{* *}$ \\
\cline { 2 - 6 } & Sig. (2-tailed) & 0.011 & & 0.000 & 0.000 \\
\hline
\end{tabular}

* Correlation is significant at the 0.05 level (2-tailed).

** Correlation is significant at the 0.01 level (2-tailed).

$\mathrm{N}: 42$ individuals.

Source: Prepared by the author.

Table 3. Influence of age on OSC, SB, SC and SP.

\begin{tabular}{|c|c|c|c|c|c|c|}
\hline \multirow{2}{*}{ Variables } & \multicolumn{2}{|c|}{ Test of Homogeneity of Variance } & \multicolumn{2}{|c|}{ Welch's Test } & \multicolumn{2}{c|}{ ANOVA } \\
\cline { 2 - 7 } & Levene's Test & Sig. & Statistic & Sig. & F & Sig. \\
\hline OSC & 1.431 & 0.251 & 0.231 & 0.795 & 0.181 & 0.835 \\
\hline SB & 4.891 & 0.013 & 0.994 & 0.386 & 1.265 & 0.294 \\
\hline SC & 2.221 & 0.944 & 0.078 & 0.925 & 0.058 & 0.944 \\
\hline SP & 2.221 & 0.047 & 2.402 & 0.114 & 3.322 & 0.047 \\
\hline
\end{tabular}

${ }^{a}$ Asymptotically $\mathrm{F}$ distributed.

Source: Prepared by the author. 
accepted, that is, statistically, independent variable work shift does not influence OSC, SB, SC nor SP.

\section{Influence of job position on OSC, SB, SC and SP}

The homogeneity test of variances and Levene's test at a significance level of $5 \%$ yielded $p$-values of $0.878,0.345,0.176$ and 0.390 for OSC, SB, SC and $\mathrm{SP}$, respectively, indicating homogeneity of variances for all variables with respect to job position (see Table 6). $P$-values of $0.107,0.218,0.389$ and 0.191 were obtained for OSC, SB, SC and SP, respectively (see Table 6), using ANOVA; a $p$-value greater than 0.05 was obtained for all cases. Consequently, $\mathrm{H}_{0}$ is accepted, that is, statistically, independent variable job position does not influence OSC, SB, SC nor SP.

\section{Influence of service time on OSC, SB, SC and SP}

The homogeneity test of variances and Levene's test at a significance level of $5 \%$ yielded $p$-values of $0.064,0.380,0.308$ and 0.105 for OSC, SB, SC and $\mathrm{SP}$, respectively, indicating homogeneity of variances for all variables with respect to service time (see Table 7). $P$-values of $0.956,0.164,0.856$ and 0.018 were obtained for OSC, SB, SC and SP, respectively (see Table 7), using ANOVA; a $p$-value greater than 0.05 was obtained for OSC, SB and SC. Consequently, $\mathrm{H}_{0}$ is accepted, that is, statistically, independent variable service time does not influence OSC, SB nor SC. For SP, a $p$-value of less than 0.05 was obtained; therefore, service time does influence SP.

\section{DISCUSSION}

The reliability of the measuring instruments was very similar to that reported by other authors. A Cronbach's alpha of 0.93 was found for occupational safety climate (OSC), which is very close to the value of 0.95 found by Zohar and Luria (2005) and higher than the 0.77 reported by Zhou and Jiang (2015). Safety behaviors (SB) obtained a value of 0.86 ; safety compliance (SC) and safety participation (SP) obtained 0.71 and 0.86 , respectively; compared to previous studies, such as that of Neal and Griffin (2006), the values obtained were 0.93 and 0.89 , respectively, with a close proximity to the SP variable.

Hypothesis $\mathrm{H}_{1}$ showed that there is a significant and positive relationship between occupational safety climate (OSC) and safety behaviors (SB), as evidenced in previous studies (Manning, Davidson \& Manning, 2005; Lyu, Hon, Chan, Wong \& Javed, 2018; Abubakar, Karadal, Bayighomog \& Merdan, 2018). Additionally, $\mathrm{H}_{2}$ and $\mathrm{H}_{3}$ also demonstrated that there is a significant and positive relationship between occupational safety climate (OSC) and safety compliance (SC) and safety participation (SP), which has been proved in a company of the steel industry in Peru and corroborates what was stated by other authors (Hon, Chan \& Yam, 2014; Zohar \& Luria, 2005; Neal \& Griffin, 2006; Lyu et al., 2018; Dahl \& Kongsvik, 2018; Mirza, Isha, Memon, Azeem \& Zahid, 2019).

Table 4. Influence of level of education on OSC, SB, SC and SP.

\begin{tabular}{|l|l|l|l|l|}
\hline \multirow{2}{*}{\multicolumn{2}{c}{ Variables }} & \multicolumn{2}{c|}{ Test of Homogeneity of Variance } & \multicolumn{2}{c|}{ ANOVA } \\
\cline { 2 - 5 } & \multicolumn{1}{c|}{ Levene's Test } & \multicolumn{1}{c|}{ Sig. } & \multicolumn{2}{c|}{ Sig. } \\
\hline OSC & 0.699 & 0.503 & 1.059 & 0.357 \\
\hline SB & 0.150 & 0.862 & 0.544 & 0.585 \\
\hline SC & 0.059 & 0.943 & 0.899 & 0.415 \\
\hline SP & 0.401 & 0.672 & 0.583 & 0.563 \\
\hline
\end{tabular}

Source: Prepared by the author.

Table 5. Influence of work shift on OSC, SB, SC and SP.

\begin{tabular}{|c|c|c|c|c|}
\hline \multirow{2}{*}{ Variables } & \multicolumn{2}{|c|}{ Test of Homogeneity of Variance } & \multicolumn{2}{|c|}{ t-test } \\
\cline { 2 - 5 } & Levene's Test & Sig. & t & 0.585 \\
\hline OSC & 0.364 & 0.550 & 0.550 & 0.693 \\
\hline SB & 3.570 & 0.066 & 0.397 & 0.394 \\
\hline SC & 8.484 & 0.006 & 0.861 & 1.000 \\
\hline
\end{tabular}

Source: Prepared by the author. 
Consequently, occupational safety climate (OSC) is related to workers' safety behaviors (SB). In this research, a significant and positive correlation between OSC, and SC and SP was found, even at the significance level of 0.01 , that is, $99 \%$ confidence that it is a true correlation. In relation to the correlation between OSC and SB, it has a significance level of 0.05 .

Using the one-factor ANOVA test, it was found that only age and service time influence workers' participation in safety. This partly supports previous findings that demonstrate the influence of socio-occupational variables on OSC and SB (Fang, Chen \& Wong, 2006; Xiong, Liang, Luo \& Fung, 2018; Wang, Mei, Liu, Zhou \& Zhang, 2019).

\section{CONCLUSIONS}

1. Occupational safety climate (OSC) is positively and significantly related to safety behaviors (SB) in a sample of workers of a steel industry company located in Peru.

2. Occupational safety climate (OSC) is directly and positively related to variable safety compliance (SC). An improvement in OSC implies an improvement in SC and vice versa.

3. Occupational safety climate (OSC) is directly and positively related to variable safety participation (SP). An improvement in OSC implies and improvement in SP.
4. No causal relationships were found between socio-occupational variables and OSC and SB regarding the workers in the operations area of this research. Nevertheless, results show behaviors or trends that might prove causality with a larger sample.

5. A causal relationship was found between socio-occupational variables age and service time of workers and safety participation (SP).

\section{REFERENCES}

[1] Abubakar, A. M., Karadal, H., Bayighomog, S. W. \& Merdan, E. (May 9, 2018). Workplace Injuries, Safety Climate and Behaviors: Application of an Artificial Neural Network. International Journal of Occupational Safety and Ergonomics. Retrieved from https://www. tandfonline.com/doi/full/10.1080/10803548.20 18.1454635.

[2] Coyle, I. R., Sleeman, S. D. \& Adams, N. (1995). Safety Climate. Journal of Safety Research, 26(4), 247-254.

[3] Dahl, Ø. \& Kongsvik, T. (2018). Safety Climate and Mindful Safety Practices in the Oil and Gas Industry. Journal of Safety Research, 64, 2936.

[4] Díaz, A. (2006). Auditoría del clima y cultura de seguridad en la empresa. (Doctoral thesis). Universidad de Valencia, Spain.

Table 6. Influence of job position on OSC, SB, SC and SP.

\begin{tabular}{|c|c|c|c|c|}
\hline \multirow{2}{*}{ Variables } & \multicolumn{2}{|c|}{ Test of Homogeneity of Variance } & \multicolumn{2}{c|}{ ANOVA } \\
\cline { 2 - 5 } & Levene's Test & Sig. & 2.372 & 0.107 \\
\hline OSC & 0.131 & 0.878 & 1.586 & 0.218 \\
\hline SB & 1.095 & 0.345 & 0.967 & 0.389 \\
\hline SC & 1.818 & 0.176 & 1.728 & 0.191 \\
\hline SP & 0.965 & 0.390 & & \\
\hline
\end{tabular}

Source: Prepared by the author.

Table 7. Influence of service time on OSC, SB, SC and SP.

\begin{tabular}{|c|c|c|c|c|}
\hline \multirow{2}{*}{ Variables } & \multicolumn{2}{|c|}{ Test of Homogeneity of Variance } & \multicolumn{2}{c|}{ ANOVA } \\
\cline { 2 - 5 } & Levene's Test & Sig. & 0.045 & 0.956 \\
\hline OSC & 2.947 & 0.064 & 1.897 & 0.164 \\
\hline SB & 0.992 & 0.380 & 0.156 & 0.856 \\
\hline SC & 1.214 & 0.308 & 4.466 & 0.018 \\
\hline
\end{tabular}

Source: Prepared by the author. 
Retrieved from https://www.tdx.cat/bitstream/ handle/10803/10188/diaz.pdf.

[5] Fang, D., Chen, Y. \& Wong, L. (2006). Safety Climate in Construction Industry: A Case Study in Hong Kong. Journal of Construction Engineering and Management, 132(6), 573-584.

[6] Griffin, M. A. \& Neal, A. (2000). Perceptions of Safety at Work: A Framework for Linking Safety Climate to Safety Performance, Knowledge, and Motivation. Journal of Occupational Health Psychology, 5(3), 347-358.

[7] Hon, C. K. H., Chan, A. P. C. \& Yam, M. C. H. (2014). Relationships Between Safety Climate and Safety Performance of Building Repair, Maintenance, Minor Alteration, and Addition (RMAA) Works. Safety Science, 65, 10-19.

[8] International Business Machines Corporation (IBM) (2019). SPSS Statistics 25.0.0. ANOVA de un factor: Opciones. Spain: IBM Knowledge Center. Retrieved from https://www.ibm.com/ support/knowledgecenter/es/SSLVMB_25.0.0/ statistics_mainhelp_ddita/spss/base/idh_ onew opt.h̆tml.

[9] Johnson, S. E. (2007). The Predictive Validity of Safety Climate. Journal of Safety Research, 38(5), 511-521.

[10] Lyu, S., Hon, C. K. H., Chan, A. P. C., Wong, F. K. W. \& Javed, A. A. (2018). Relationships among Safety Climate, Safety Behavior, and Safety Outcomes for Ethnic Minority Construction Workers. International Journal of Environmental Research and Public Health, 15(484), 1-16.

[11] Manning, M. L., Davidson, M. \& Manning, R. L. (2005). Measuring Tourism and Hospitality Employee Workplace Perceptions. International Journal of Hospitality Management, 24(1), 75-90.

[12] Melià, J. L. \& Sesé, A. (1999). La medida del clima de seguridad y salud laboral. Anales de Psicología, 15(2), 269-289.

[13] Mirza, M., Isha, A., Memon, M., Azeem, S. \& Zahid, M. (15 de mayo de 2019). Psychosocial Safety Climate, Safety Compliance and Safety Participation: The Mediating Role of Psychological Distress. Journal of Management \& Organization. Retrieved from https://www.cambridge.org/core/journals/ journal-of-management-and-organization/ article/psychosocial-safety-climate-safetycompliance-and-safety-participation-themediating-role-of-psychological-distress/942B D88321E73482B4A4D99A3D2D0D9A.
[14] Neal, A. \& Griffin, M. A. (2004). Safety Climate and Safety at Work. In J. Barling \& M. R. Frone (Eds.), The Psychology of Workplace Safety (pp. 15-34). Washington D. C., United States: American Psychological Association.

[15] Neal, A. \& Griffin, M. A. (2006). A Study of the Lagged Relationships Among Safety Climate, Safety Motivation, Safety Behaviour and Accidents at the Individual and Groups Levels. Journal of Applied Psychology, 91(4), 946-953.

[16] Peiró, J. M. (1984). Psicología de la organización. Madrid, Spain: Universidad Nacional de Educación a Distancia (UNED).

[17] Ruscio, J. (2008). Constructing Confidence Intervals for Spearman's Rank Correlation with Ordinal Data: A Simulation Study Comparing Analytic and Bootstrap Methods. Journal of Modern Applied Statistical Methods, 7(2), 416434.

[18] Sawilowsky, S. \& Blair, R. C. (1992). A More Realistic Look at the Robustness and Type II Error Probabilities of the Test to Departures from Population Normality. Psychological Bulletin, 111(2), 352-360.

[19] Schmider, E., Ziegler, M., Danay, E., Beyer, L. \& Bühner, M. (2010). Is It Really Robust? Reinvestigating the Robustness of ANOVA Against Violations of the Normal Distribution Assumption. Methodology: European Journal of Research Methods for the Behavioral and Social Sciences, 6(4), 147-151.

[20] Stonehouse, J. M. \& Forrester, G. J. (1998). Robustness of the $t$ and $U$ tests under combined assumption violations. Journal of Applied Statistics, 25(1), 63-74.

[21] Wang, Q., Mei, Q., Liu, S., Zhou, Q. \& Zhang, J. (2019). Demographic Differences in Safety Proactivity Behaviors and Safety Management in Chinese Small-Scale Enterprises. Safety Science, 120, 179-184.

[22] Warner, P. (2010). Quantifying Association in Ordinal Data. Journal of Family Planning and Reproductive Health Care, 36(2), 83-85.

[23] Xiong, C., Liang, K., Luo, H. \& Fung, I. W. H. (2018). Identification of Safety-Related Opinion Leaders among Construction Workers: Evidence from Scaffolders of Metro Construction in Wuhan, China. International Journal of Environmental Research and Public Health, 15(2176), 1-17. 
[24] Zhou, F. \& Jiang, C. (2015). Leader-Member Exchange and Employees' Safety Behavior: The Moderating Effect of Safety Climate. Procedia Manufacturing, 3, 5014-5021.

[25] Zohar, D. (1980). Safety Climate in Industrial Organizations: Theoretical and Applied Implications. Journal of Applied Psychology, 65(1), 96-102.
[26] Zohar, D. \& Luria, G. (2005). A Multilevel Model of Safety Climate: Cross-Level Relationships Between Organization and Group-Level Climates. Journal of Applied Psychology, 90(4), 616-628. 